\title{
EO Performances of Flexible TN-LCD using in-situ Ultraviolet Exposure during Imidization of Polyimide on the Polymer Film
}

\author{
Hyun-Chan Moon, Jeoung-Yeon Hwang, Whee-Won Lee, and Dae-Shik Seo ${ }^{\mathrm{a}}$ \\ Department of Electrical \& Electronic Engineering, Yonsei University, \\ 134 Shinchon-dong, Seodaemoon-gu, Seoul 120-749, Korea \\ âE-mail : dsseo@yonsei.ac.kr
}

(Received April 8 2005, Accepted June 13 2005)

\begin{abstract}
We have investigated the generation of pretilt angle for a nematic liquid crystal (NLC) alignment with in-situ photoalignment method on polyimide (PI) surfaces using polymer films. Especially, we studied in-situ photoalignment changing heating temperature from $50{ }^{\circ} \mathrm{C}$ to $120{ }^{\circ} \mathrm{C}$ on the polymer film. The LC aligning capabilities and pretilt angle on the polymer substrates were better than those on the glass substrate using in-situ photoalignment method. It is considered that this increase in pretilt angle may be attributed to the roughness of the micro-groove substrate induced by the in-situ photoalignment. As temperature of heated subtrate and UV exposure time increase, pretilt angle of the cell used polymer film increased. It is considered that the heating temperature of substrate is attributed to generate pretilt angle. Also, electro-optical performances of the in-situ photoaligned TN cell using the polymer substrate are almost the same as that of the TN cell using the glass substrate.
\end{abstract}

Keywords : in-situ photoalignment method, Pretilt angle, Nematic liquid crystal, Polymer film

\section{INTRODUCTION}

Flexible display based on polymer substrates for liquid crystal displays (LCDs) has several advantages compared to display using glass substrates. They exhibit only $1 / 6$ of the weight of glass substrate. Flexible displays are virtually unbreakable and their flexibility allows the designer to do elegant styling[1].

Flexible display has many problems yet in spite of that fact the display has these advantages; for example, the first, polymer films have low thermal stability below $180^{\circ} \mathrm{C}$. The second, a liquid crystal (LC) alignment on polymer film followed by cloth rubbing has several problems[2,3]. Most general polyimide (PI) layer need high curing temperature to form alignment layer. However, polymer films are difficult to apply this manufacturing line because polymer films are thermally unstable. Also, the control of rubbing strengths is difficult because film thickness is thin like $0.2 \mathrm{~mm}$.

Therefore, a rubbing-free method for LC alignment is required for the fabrication of a flexible LCD. Many researchers have reported a rubbing-free method for LC alignment[4,5]. Most recently, the LC alignment and pretilt angle generation using the in-situ photoalignment method on a PI surface for homogeneous alignment have been reported[6]. The in-situ photoalignment method produces a higher thermal stability of LC alignment than that produced using the conventional photoalignment method. We have investigated in-situ photoalignment method on the glass substrate[7,8]. However, the effect of the nemtic liquid crystal (NLC) pretilt angle and electro-optical (EO) performance by using the in-situ photoalignment method on the polymer film have not been reported yet.

In this study, we studied LC alignment effects and the electro-optical (EO) characteristics of in-situ photoaligned TN cell on the polymer film.

\section{EXPERIMENTAL}

Figure 1 shows the chemical structure of the polyimide (PI) used in this study (from Nissan Chemical industries). We used a polycarbonate (PC) film $(200 \mu \mathrm{m})$ as the substrate. In the conventional photoalignment method, the polymers were soft-baked at $80{ }^{\circ} \mathrm{C}$ for 10 min and baked at $120{ }^{\circ} \mathrm{C}$ for $\mathrm{lh}$. The thickness of the PI layer was set at $500 \AA$. The UV exposure system is shown in Fig. 2. The UV source was a $1000 \mathrm{~W}$ Xe lamp. In the in-situ photoalignment method, polymers were exposed to obliquely polarized UV during imidization of 


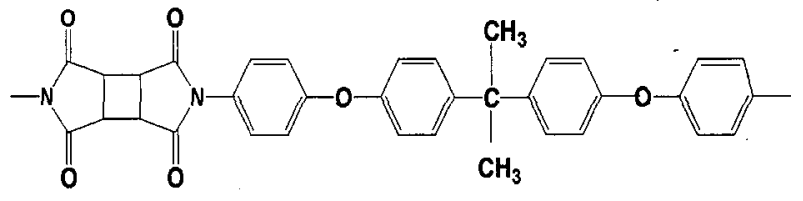

Fig. 1. Chemical structure of the polymer.
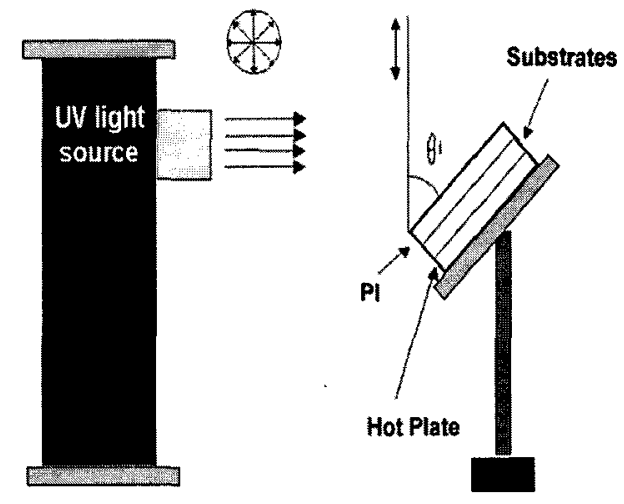

Fig. 2. UV exposure system used.

polyimide at $50 \sim 120{ }^{\circ} \mathrm{C}$. To measure tilt angle, the cell was fabricated as a sandwich type, and the thickness of the cell was $60 \mu \mathrm{m}$. A nematic liquid crystal (NLC) $\left(\mathrm{T}_{\mathrm{c}}=\right.$ $72{ }^{\circ} \mathrm{C}, \triangle \varepsilon=8.2$, from Merck Co.) of positive type NLC ( $\Delta \varepsilon=8.2$, from Merck Co.) was injected to the isotropic phase. The other is the twisted nematic (TN) cell, which is used for Electro-Optical measurement. The thickness of the liquid crystal cells for TN cell was $5 \mu \mathrm{m}$. The crystal rotation method was used to measure pretilt angle (from Autronic Co.). LC alignment effects were observed by using a polarized microscope. In addition Voltage-Transmittance and response time characteristics of in-situ photoaligned TN cell were measured by LCD EOMS (Electro-optical Measurement, from Sesim Photonics Technology) equipment.

\section{RESULTS AND DISCUSSION}

Figures 3 show the microphotographs of aligned NLC using the conventional and in-situ photoalignment method with obliquely non-polarized UV exposure of $45^{\circ}$ on the PI surfaces using polymer film. The large disclinations of NLC were observed by conventional photoalignment method. However, good alignment with in-situ photoalignment was obtained. It is considered that the better aligning capability of NLC is attributed to the high temperature of polymer substrate. The LC aligning capability of in-situ photoalignment method is better than that of the conventional alignment method.

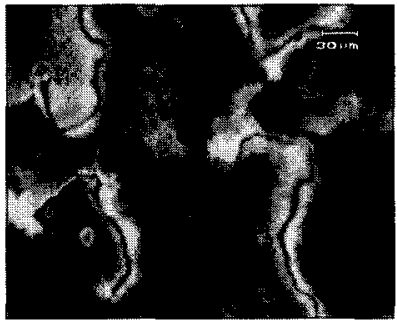

(a) Conventional photoalignment method

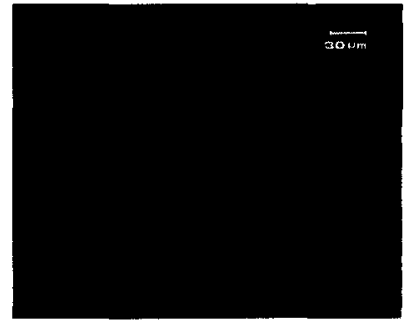

(b) In-situ photoalignment method
Fig. 3. Microphotographs of photoaligned LC cell using general and in-situ photoalignment method on the glass and plastic substrate (incrossed Nicols).

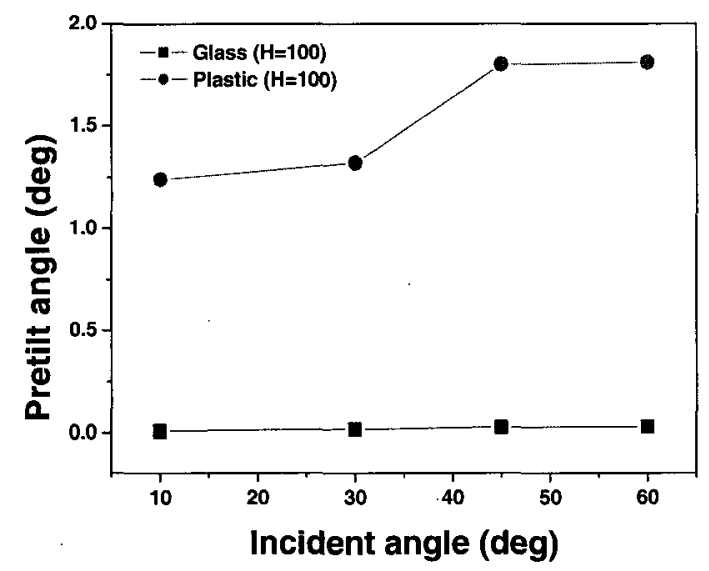

Fig. 4. Generation of pretilt angles in NLC on the glass and plastic substrate as a function of non-polarized insitu UV incident angle.

The pretilt angles in NLC with non-polarized in-situ UV exposure on the glass and polymer substrate as a function of incident angle are shown in Fig. 4. The high pretilt angle generated in NLC was observed by in-situ UV exposure on the polymer substrate for $1 \mathrm{~min}$. The pretilt angle of NLC increases with increasing UV exposure angle. However, the low pretilt angle in NLC on the glass substrate was measured below $1^{\circ}$ for all UV exposure angle. Therefore, the pretilt angle on the polymer film is higher than that on the glass using in-situ photoalignment method. The surface roughness of the polymer substrate is higher than that of the glass substrate. It is considered that this increase in pretilt angle may be attributed to the roughness of the microgroove substrate induced by the in-situ photoalignment. These results demonstrate the theory that the topographical structure of the alignment layer contributes to the LC alignment[9].

Figure 5 shows NLC pretilt angles with obliquely nonpolarized in-situ UV exposure of the plastic substrate as 


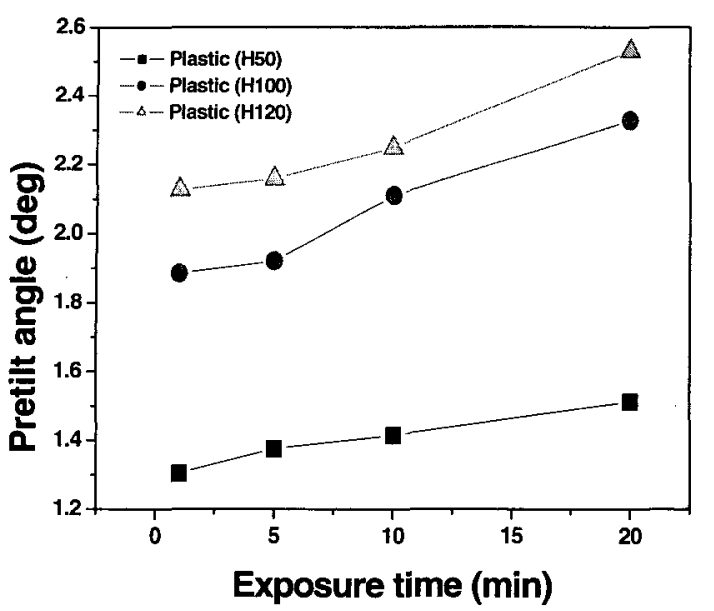

Fig. 5. Generation of pretilt angles in NLC as a function of substrate's heating temperature and UV exposure time on the in-situ photoalignment method.

a function of UV exposure time and heating temperature. The pretilt angle of NLC increases with increasing UV exposure time and substrate's heating temperature. Moreover, increasing of heat temperature that is imposed on polymer substrate can give important influence on moving molecules and surface topography of the microroughness of the substrate. So, it is considered that the heating temperature of substrate is attributed to generate pretilt angle. These results in polymer substrate show that heating effect on the PI surface can promote photodissociation action. Therefore, we suggest that insitu alignment method contribute to the high pretilt angle for NLC on the polymer substrate.

Figure 6 shows the voltage-transmittance (V-T) characteristics of the in-situ photoaligned TN cell on glass and polymer substrates when heating temperature of two substrates is $100{ }^{\circ} \mathrm{C}$. All stable curves of the insitu photoaligned TN cell on glass and polymer substrates were achieved; The TN cells show a good transmission of light as a function of applied voltage across TN cells made by glass and polymer films, for using liquid crystal display. Therefore, V-T characteristics of the in-situ photoaligned $\mathrm{TN}$ cell using the polymer substrate are almost the same as those of the insitu photoaligned TN cell using the glass substrate.

Figure 7 shows the response time (RT) characteristics of the in-situ photoaligned TN cell on glass and polymer substrates when heating temperature of two substrates is $100{ }^{\circ} \mathrm{C}$. The two RT curves have a difference as shown Fig. 7. The response time-transmittance characteristics of the in-situ TN cell with the polymer film exhibited a more stable quality than those of the in-situ photoaligned TN cell with the glass substrate. Especially, RT curve of in-situ TN cell with the glass substrate showed big back-

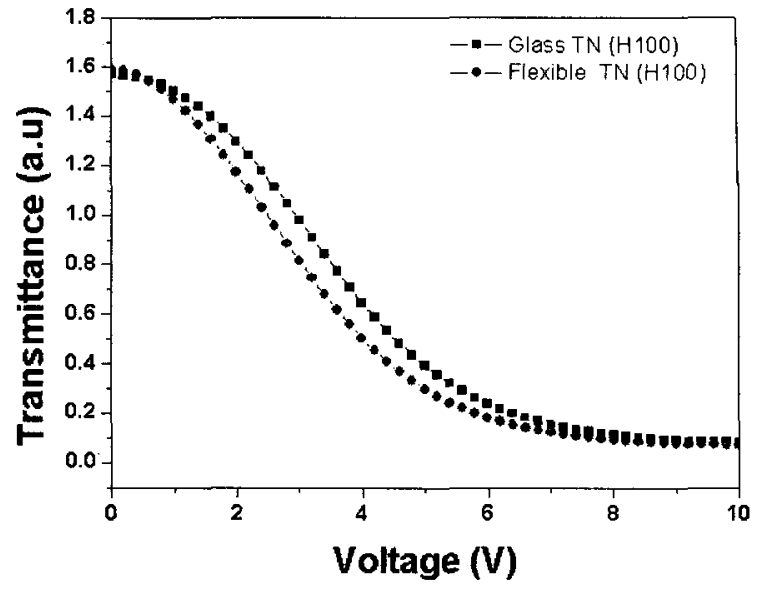

Fig. 6. The voltage-transmittance (V-T) characteristics of the in-situ aligned $\mathrm{TN}$ cell on glass and polymer substrates when heating temperature of two substrates is $100{ }^{\circ} \mathrm{C}$.

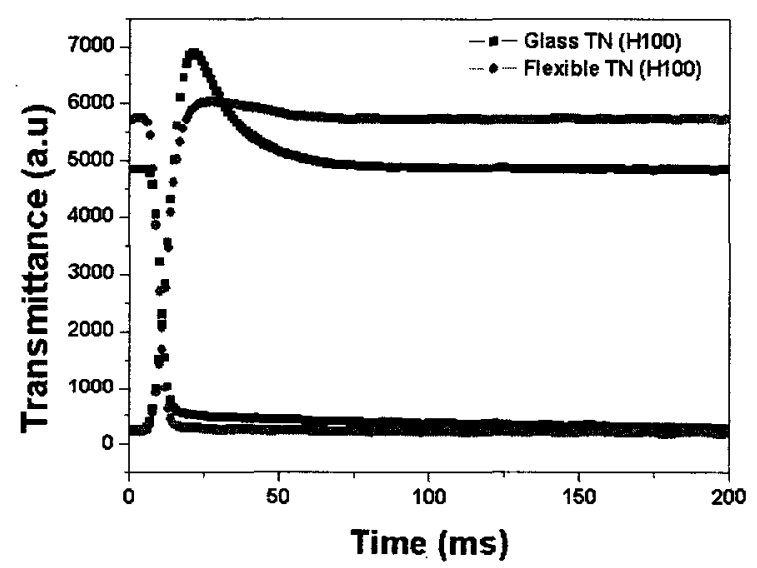

Fig. 7. The response time (RT) characteristics of the insitu aligned TN cell on glass and polymer substrates when heating temperature of two substrates is $100{ }^{\circ} \mathrm{C}$.

flow bounces. It is considered that LC aligning capability generated film by in-situ photoalignment method with the polymer film is stronger than that by the in-situ photoalignment method with the glass substrate. Also, the response time of the in-situ photoaligned TN cell using the polymer substrate was measured to be about 15 ms.

When a polymer film is used in the in-situ photoalignment method, the pretilt angle characteristics were higher than those obtained when the glass substrates were used, and the electro-optical performances of the TN cell using the polymer substrate is almost the same as that of the TN cell using the glass substrate. 


\section{CONCLUSION}

In conclusion, $\mathrm{LC}$ alignment effects and generation of pretilt angles with in-situ photoalignment irradiation changing heating temperature from $50{ }^{\circ} \mathrm{C}$ to $120{ }^{\circ} \mathrm{C}$ on the polymer film were investigated, and the EO performances of the in-situ photoaligned TN-LCD with polymer film were studied. LC aligning capability with the polymer substrate was obtained the better than that on glass substrate using in-situ photoalignment method. The pretilt angles of the polymer substrates by in-situ photoalignment method were higher than those of the glass substrate by in-situ photoalignment method. As temperature of heated substrate and UV exposure time increase, pretilt angles of the cell used polymer film increased. It is considered that the heating temperature of substrate is attributed to generate pretilt angle. In the insitu photoalignment method, the EO performances of the $\mathrm{TN}$ cell using the polymer substrate is almost the same as those of the TN cell using the glass substrate.

\section{ACKNOWLEDGMENTS}

This work was supported by National Research Laboratory program (M1-0203-00-0008) the Ministry of Information \& Communications of Korea under the Information Technology Research Center (ITRC) Program.

\section{REFERENCES}

[1] E. Lueder, "Liquid crystal display", John Wiley \& Sons Ltd, p. 307, 2001.

[2] D. S. Seo, K. Araya, N. Yoshida, M. Nishikawa, Y.
Yabe, and S. Kobayashi, "Effect of the polymer tilt angle for generation of pretilt angle in nematic liquid crystal on rubbed polyimide surfaces", Jpn. J. Appl. Phys., Vol. 34, No. 4B, p. 503, 1995.

[3] H. Matsuda, D. S. Seo, N. Yoshida, K. Fujibayashi, S. Kobayashi, and Y. Yabe, "Estimation of the static electricity and optical retardation produced by the rubbing polyimide and polyamide films with different fabrics", Mol. Cryst. \& Liq. Cryst., Vol. 264 p. 23, 1995.

[4] J. Y. Hwang, D. S. Seo, O. Kwon, and D. H. Suh, "Electro-optical characteristics of photo-aligned TNLCD on PM4Ch surfaces", Liq. Cryst., Vol. 27, No. 8 p. $1045,2000$.

[5] D. S. Seo and J. Y. Hwang, "Liquid crystal aligning capabilities using a new photo-dimerization method on a poly(4'-methacryloyloxy chalcone) surface", Jpn J. Appl. Phys., Vol. 39, No. 8A, p. L816, 2000.

[6] J. H. Kim, B. R. Acharya, Kumar, and K. R. Ha, "A method for liquid crystal alignment using in situ ultraviolet exposure during imidization of polyimide" Appl. Phys. Lett., Vol. 23, No. 4, p. 3372, 1998.

[7] J. Y. Hwang and D. S. Seo, "Control of high pretilt angle for nematic liquid crystal using in situ photoalignment method on homeotropic alignment layer", Jpn. J. Appl. Phys., Vol. 40, No. 6A, p. 4160, 2001.

[8] J. Y. Hwang, K. S. Park, D. H. Suh, and D. S. Seo, "EO performances of the photoaligned VA-LCD on the polynorbornene derivative layers", Mol. Cryst. \& Liq. Cryst., Vol. 410, p. 275, 2004.

[9] J. Y. Hwang, K. H. Nam, J. H. Kim, D. S. Seo, and D. H. Suh, "Characterization and alignment properties on polyimide surface using polymer films for flexible liquid crystal displays", Jpn. J. Appl. Phys., Vol. 43, No. 12, p. 8179, 2004. 